\title{
Handling Conflicts to Test Transport Protocol's Parallel Routing on a Vehicle Gateway System
}

\author{
Hassan Mohammad \\ MBtech Group GmbH \& Co. KGaA \\ Sindelfingen 71063, Germany \\ Email:hassan.mohammad@mbtech-group.com
}

\author{
Muhammad Shamoon Saleem \\ Ingolstadt University of Applied Sciences \\ Ingolstadt 85049, Germany \\ Email:ia2632@thi.de
}

\begin{abstract}
This paper addresses the issue of verifying transport protocol's parallel routing functionality on a vehicle gateway system. The focus of the paper is to construct a conflict-free input parameter model for testing this functionality. The input parameter model shall support the reduction of combinations to be tested and serves as a basis for automatic test case generation from a large space of input parameters. In the proposed approach, defined similarity criteria are used to cluster system input parameters represented as transport protocol routing instances into groups which stimulate similar behavior in the gateway when transport protocol routing is established. Subsequently, the two conflict-handling methods sub-models and avoid are utilized to prohibit invalid combinations of transport protocol routing instances. The proposed approach is applied on a complex example of real gateway with five buses, 390 transport protocol routing instances and diverse conflicts to illustrate its applicability.
\end{abstract}

Index Terms-Transport Protocol's Parallel Routing, Input Parameter Model (IPM), Conflict Handling

\section{INTRODUCTION}

$\mathbf{T}$ ODAY'S vehicles Electric/Electronic (E/E) systems are designed as distributed systems in order to overcome the increasing complexity and to meet the diversity of requirements such as performance, comfort, safety and costs. In a vehicle distributed system, gateways are indispensable. They enable Electric Control Units (ECUs) within connected networks to interchange information necessary for accomplishing specified functionalities. During information interchange, the gateway routes data between its connected networks although they work on different communication protocols.

In a vehicle gateway system, routing data packets which are larger than a single frame of the corresponding network communication protocol is carried out with the help of transport protocol implementations and such type of routing is called TP routing. Since TP data packets are mostly large in size (flash data for example) and routing of such large data packets requires longer duration, modern gateways support TP parallel routing where multiple TP routing instances are established in parallel over the gateway in order to save time and resources, e.g., flashing multiple ECUs in parallel.

Verifying TP parallel routing of a gateway system is not a trivial problem, since a large number of possible combinations of communicating ECUs can be built for test case selection

This work was supported by MBtech Group GmbH \& Co. KGaA (the combinatorial explosion problem). Furthermore, in case of established TP parallel routing, different types of conflicts between ECUs need to be handled.

Combination strategies [1] are test case selection methods that focus on solving the combinatorial explosion problem raised while testing the interactions between system input parameter values by defining coverage criteria to satisfy. However, the problem in the case of verifying TP parallel routing on a vehicle gateway system is different than described problem of combination test strategies (see II-B). Hence, new techniques need to be defined.

In combination strategies, Input Parameter Models (IPMs) [2] [3] [4] [5] are essential. They represent the System Under Test (SUT) on an abstract level. Mostly, IPMs contain conflicts which must be resolved. A conflict in an IPM is due to an invalid combination of input parameter values and hence this combination must be omitted or avoided while generating test cases. Diverse conflict handling strategies such as sub-models and avoid have been suggested in literature [6] to overcome conflicts in IPMs.

This paper suggests an approach to build a conflict-free IPM used to test TP parallel routing on a vehicle gateway system. The IPM is utilized in a recursive way for test case selection, generation and execution in order to overcome the combinatorial explosion problem.

The remainder of this paper is organized as follows. Section II gives background information on terminology and the combinatorial explosion problem of testing TP parallel routing. An IPM along with conflict handling mechanisms are presented in details in section III. In section IV, the suggested IPM along with described conflict handling mechanisms are utilized in a method to reduce test suite size. The complete methodology is applied on a complex example of real gateway in section V. Section VI discusses the approach and section VII concludes the paper.

\section{BACKGROUND}

\section{A. Vehicle Bus Communication Systems}

Generally, different bus communication systems are utilized in $\mathrm{E} / \mathrm{E}$ system. In this subsection, two common types of automotive buses are briefly explained. 
1) CAN Communication Bus: Controller Area Network (CAN) bus system was originally invented to reduce the wiring harness in automotive E/E systems by developing a serial communication protocol. CAN has the capability to connect multiple ECUs directly to one medium, which leads to better management of the system complexity and the reduction of manufacturing costs. CAN protocol is an asynchronous serial protocol that enables real time communication. In CAN, the medium access is based on the concept of Carrier Sense Multiple Access/Collision Detection (CSMA/CD).

2) FlexRay Communication Bus: FlexRay was developed to deliver deterministic, fault-tolerant and high-speed communication bus required for $\mathrm{x}$-by-wire applications such as steer-by-wire and break-by-wire. These applications demand more safety, performance and reliability than provided by CAN. FlexRay unifies time- and dynamic event-triggering mechanisms in one protocol. The communication in FlexRay is based on the Time Devision Multiple Access (TDMA) and Flexible Time Division Multiple Access (FTDMA) schemes of networking.

\section{B. Terminology}

The vehicle gateway is part of a distributed network system. It is a special ECU that has multiple communication channels used to communicate with other ECUs in the network in order to route data between them. Communication channels of the gateway are mostly heterogeneous in respect of characteristics and behavior. Generally, a number of ECUs $(u)$ can exchange data over the gateway in predefined fashions. Each fashion is characterized through a set of gateway configuration parameters. These are required by the gateway to establish routing between communicating ECUs connected to different communication channels. For TP routing over the gateway, following definitions are considered:

A TP_Routing_Fashion describes a possible routing behavior of TP data and is characterized through a particular set of gateway configuration parameters. An example of a $T P \_$Routing_Fashion $F$ with $P$ parameters shall be described (1) (see [7] for configuration parameters of CAN TP).

$$
\text { TP_Routing_Fashion } P_{F}=\left\{P_{F_{1}}, P_{F_{2}}, \ldots, P_{F_{P}}\right\}
$$

A TP_Connection_Channel is an instance of a TP_Routing_Fashion. It has the same set of gateway configuration parameters and is utilized to route TP data between respective ECUs. A TP_Connection_Channel ${ }_{x}$ in the TP_Routing_Fashion ${ }_{F}$ shall be described (2).

$$
T P \_C o n n e c t i o n \_C h a n n e l_{x}=\left\{P_{F_{1} x}, P_{F_{2} x}, \ldots, P_{F_{P} x}\right\}
$$

The gateway has a number of configured TP_Connection_Channels for connected ECUs utilized to establish TP routing in different scenarios. Examples of TP scenarios are flashing and Onboard Diagnostic (OBD). TP_Routing_Scenarios shall be described as a group of $s$ scenarios (3).

$$
T P \_R o u t i n g \_S c e n a r i o s=\left\{S_{1}, S_{2}, \ldots, S_{s}\right\}
$$

A TP_Routing_Instance is a relationship between a specific TP_Connection_Channel and a possible TP_Routing_Scenario. An example of a TP_Routing_Instance ${ }_{x}$ shall be described (4).

$$
\text { TP_Routing_Instance } x=\left\{P_{F_{1 x}}, P_{F_{2 x}}, \ldots, P_{F_{P x}}, S_{x}\right\}
$$

The gateway can be configured to serve $y$ TP_Routing_Instances in parallel. The number of parallel $T P \_$Routing_Instances " $y$ " is a configuration parameter which needs to be verified. In the case of errors, the next determined " $y$ " should be verified.

\section{The Combinatorial Explosion Problem of Testing TP Par- allel Routing}

The combinatorial explosion problem mentioned in literature [8] [9] [10] shall be explained as in the following example:

Assume a distributed system consisting of a central unit interacting over communication channels with $u$ units of the network $U_{1}, U_{2}, \ldots, U_{u}$. Each unit $U_{i}$ uses a defined parameter $p_{i}$ for communication. The parameter $p_{i}$ shall have $v_{i}$ possible configuration values. By assuming that configuration values of parameters are independent from each other, the number of possibilities in which the system can be configured would be $v_{1} * v_{2} * \ldots * v_{u}$. If each possible configuration requires $c$ test cases to verify it, the number of test cases for exhaustive test would be $c * v_{1} * v_{2} * \ldots * v_{u}$. In a nontrivial software system, the values of $u$ and $v_{i}$ are large which leads to a huge number of possible combinations of parameter values.

Related to testing TP parallel routing, the goal of test is to measure the performance of the gateway to handle parallel TP_Routing_Instances. The problem is more complex because:

- System input parameters are TP_Routing_Instances where each consists of a set of configuration parameters.

- The number of system input parameters is not fixed. It can be different for every new release of the system.

- System input parameters include also timing parameters where the interactions are difficult to resolve.

- The number of parallel TP_Routing_Instances " $y$ ", which is also a configuration parameter, is used to build possible combinations to be tested. Combinations are any $y$ elements from the system input parameter set. In case of errors, one of the goals is to determine the next " $y$ " and verify it (performance measurement).

- In TP parallel routing, each additional instance will consume resources of the system and may lead to errors. Hence, it is not only a specific combination of TP_Routing_Instances which can affect the behavior and may reveal errors, but also the number of included $T P \_$Routing_Instances and their values.

To verify " $y$ " parallel routing of TP_Routing_Instances and determine the next $" y "$ in case of errors, all possible combinations from 1 to $y$ of TP_Routing_Instances should be included at least once in test cases. This results in a number 
of combinations to be tested which can be calculated using equation(5).

$$
\begin{aligned}
X & =\frac{u !}{y !(u-y) !} \cdot y^{s}+\frac{u !}{(y-1) !(u-(y-1)) !} \cdot(y-1)^{s} \\
& +\ldots+\frac{u !}{1 !(u-1) !} \cdot 1
\end{aligned}
$$

The equation (5) calculates the sum of all possible combinations for a number of ECUs $(u)$ communicating in $T P \_$Routing_Scenarios $(s)$, where the selected number of ECUs in each term varies from $y$ to 1 .

Even for a simple system, the duration of testing for all these combinations is too high and therefore not acceptable.

\section{INPUT PARAMETER MODEL AND CONFLICT HANDLING}

A combination test strategy consists generally of two steps:

1) Building a suitable IPM

2) Selecting combinations of parameter values from IPM to satisfy defined coverage criteria.

In this paper, the proposed IPM shall be used to test TP parallel routing in a recursive approach, i.e., one combination is constructed at a time. Subsequently, a test case is generated for that combination and executed. This procedure is then repeated until satisfying the defined coverage criterion. The decision on using a recursive selection and execution approach has two reasons:

1) Information from executed test cases is collected and can be used to reduce the number of combinations for the successive test cases.

2) Gained information from executed test cases is utilized to determine the worst case combinations.

In order to build a suitable conflict-free IPM, conflicts need to be defined. In a TP parallel routing, three types of conflicts are stated:

1) Type $_{\mathrm{A}}$-Conflicts: Conflicts between $T P \_$Routing_Scenarios. These are constraints describing $T P \_$Routing_Scenarios not practiced in parallel.

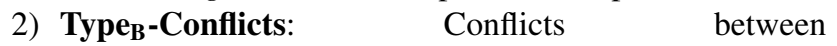
TP_Connection_Channels. These are constraints describing TP_Connection_Channels not allowed to be combined in parallel.

3) Type ${ }_{C}-$ Conflicts: Conflicts because of configuration parameters. These are constraints describing known or desired capabilities of the SUT.

The following example explains raised conflicts in testing TP parallel routing on a vehicle gateway system:

Assume a gateway having six TP_Routing_Instances in TP_Routing_Fashions $\left(F_{x}\right.$ and $\left.F_{y}\right)$ used to route TP data between connected ECUs as following:

$T P_{-}$Routing_Instance $1=\left(P_{F_{x 11}}, P_{F_{x 21}}, P_{F_{x 31}}, P_{F_{x 41}}, S_{1}\right)$ $T P_{\text {Routing_Instance }}=\left(P_{F_{x 12}}, P_{F_{x 22}}, P_{F_{x 32}}, P_{F_{x 42}}, S_{2}\right)$ $T P_{-}$Routing_Instance $3=\left(P_{F_{x 13}}, P_{F_{x 23}}, P_{F_{x 33}}, P_{F_{x 43}}, S_{1}\right)$ $T P \_$Routing_Instance $4=\left(P_{F_{x 14}}, P_{F_{x 24}}, P_{F_{x 34}}, P_{F_{x 44}}, S_{2}\right)$
$T P \_$Routing_Instance $5=\left(P_{F_{y 15}}, P_{F_{y 25}}, P_{F_{y 35}}, S_{1}\right)$

$T P \_$Routing_Instance $6=\left(P_{F_{y 16}}, P_{F_{y 26}}, P_{F_{y 36}}, S_{2}\right)$

Assume also that the gateway supports two TP_Routing_Instances in parallel. Then, following examples explain the three types of conflicts:

- Type $_{\mathrm{A}}$-Conflicts: TP_Routing_Scenario $S_{1}$ and $T P \_$Routing_Scenario $S_{2}$ are non-combinable. That is, a combination of TP_Routing_Instance ${ }_{1}$ and $T P \_$Routing_Instance 2 is for example an invalid combination.

- Type $_{\mathbf{B}}-$ Conflicts: TP_Connection_Channel 2 and TP_Connection_Channel 4 are non-combinable, i.e., a combination of TP_Routing_Instance 2 and $T P \_$Routing_Instance 4 is an invalid combination.

- Type $_{\mathbf{C}}$-Conflicts: Maximum of two TP_Routing_Instances are combinable, i.e., all combinations of more than two TP_Routing_Instances are invalid.

As discussed in the previous section, a TP_Routing_Instance of gateway is a relationship between $T P \_C o n n e c t i o n \_C h a n n e l$ and possible TP_Routing_Scenario. $T P \_$Routing_Instances are input parameter values required to build a conflict-free IPM for testing. To achieve building a conflict-free IPM which supports the reduction of combinations in a recursive approach, following steps are required:

1) Collecting and extending TP_Routing_Instances based on similarity criteria.

2) Handling conflicts.

\section{A. Collecting and Extending TP_Routing_Instances based on Similarity Criteria}

In collecting TP_Routing_Instances, instances that stimulate similar behavior in the gateway are clustered into groups. Two TP_Routing_Instances are said to be similar if and only if they have the same values for all related parameters such as routing parameters, network relationship parameters and mapped TP_Routing_Instance. Creating groups of similar $T P \_$Routing_Instances helps in reducing the number of combinations required for testing. The resulting combination from formulating groups are defined as "reduced combinations".

Following example explains reduction achieved after groups are constructed.

Assume that the SUT has 4 TP_Routing_Instances A, B, $\mathrm{C}$ and D (see Fig. 1) and it is configured to support 2 $T P \_$Routing_Instances in parallel. The number of possible combinations of 2 instances out of 4 would be 6 (the order has no effect). If TP_Routing_Instances A,B and C,D are similar to each other, then groups can be constructed based on similarity criteria such that Group $_{1}$ consists of instances A and B, whereas Group 2 consists of instances C and D. After grouping, the number of combinations could be rather reduced from 6 to 3, because all other possible combinations would resemble a similar behavior, i.e., combinations of instances 


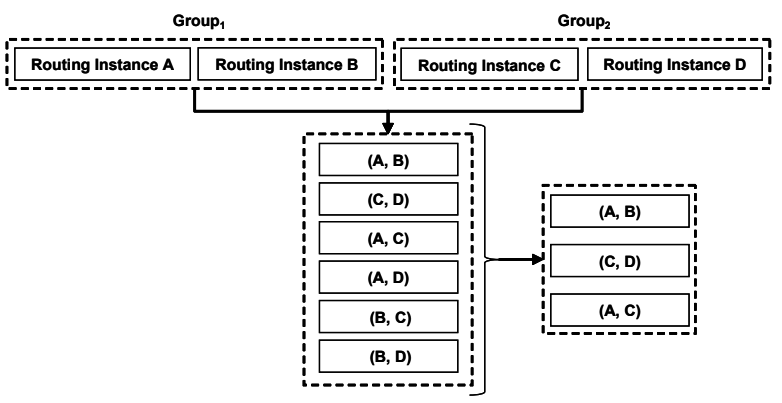

Fig. 1. Advantages of Building Similar Groups

$(\mathrm{A}, \mathrm{C}),(\mathrm{A}, \mathrm{D}),(\mathrm{B}, \mathrm{C})$ and $(\mathrm{B}, \mathrm{D})$ are all similar and can be replaced by only one substitute $(A, C)$. Therefore $(A, B),(C, D)$ and $(\mathrm{A}, \mathrm{C})$ are the "reduced combinations".

In extending TP_Routing_Instances, Similarity Numbers and Stress Factors are assigned to constructed groups. The same Similarity Number will be assigned to groups with $T P \_$Routing_Instances having the same routing parameters, the same TP_Routing_Scenario and the same characteristics for network relationship. Concerned network characteristics are the protocol type and channel bandwidth. The Stress Factor is calculated based on aspects such as processing time, memory usage, channel bandwidth and other channel specific aspects. Stress Factors shall be assigned during test case execution. Similarity Numbers and Stress Factors are required for reducing combinations during testing. This will be discussed later in details. The basic idea to resolve the combinatorial explosion problem is by using "reduced combinations" formulated from constructed groups based on similarity criteria.

\section{B. Conflict Handling}

In [2], four different methods for handling conflicts in IPMs were investigated. The result of the study was that the avoid method is best suited with respect to size of test suite if it can be utilized. To handle conflicts with the avoid method; a procedure is integrated in the test case selection algorithm to prohibit choosing of conflicting combinations. Another method mentioned in the study is the sub-models method, in which conflicts are removed by splitting the IPM into multiple smaller conflict-free IPMs used separately to generate test cases. In this paper, a combination of these two conflict handling methods is utilized to handle the defined conflicts for testing TP parallel routing.

1) Type ${ }_{A}$-Conflict Handling: Since constructed groups possess TP_Routing_Instances similar to each other in all assigned parameters, TP_Routing_Instances of each group will have the same attached TP_Routing_Scenario. To handle conflicts existing between TP_Routing_Scenarios, the sub-models method is utilized. The TP_Routing_Scenario is used to split IPM into sub-IPMs which are Type $_{\mathrm{A}}-$ Conflict-free. The resulted sub-IPMs shall have no combinable TP_Routing_Instances among each other. This step can be completely achieved before executing any test case, i.e., it is not part of the recursive mechanism. Subsequently, resulted sub-IPMs are processed successively in a recursive methodology.

2) Type $_{B}$-Conflict Handling: As described before, a TP_Routing_Instance is a relationship between a TP_Connection_Channel and a TP_Routing_Scenario. In order to handle conflicts between TP_Connection_Channels, the avoid method is utilized. To implement the avoid method, two reserved parameters, i.e., "Token" and "Include Times", are attached to individual TP_Routing_Instances. The value of the first parameter "Token" is used to decide whether a $T P \_$Routing_Instance is allowed to be included in the next combination or not. The Token parameter can have one of the following values:

- 0: Related TP_Routing_Instance having no Type ${ }_{\mathrm{B}}-$ conflicts and is allowed to be included in a combination.

- 1: Related TP_Routing_Instance having Type $_{\mathrm{B}}$-conflicts and is allowed to be included in the next combination.

- 2: Related TP_Routing_Instance having Type B $_{\mathrm{B}}$-conflicts and is not allowed to be included in the next combination.

The second parameter "Include Times" is used to hold the number of times a TP_Routing_Instance is included in constructed combinations. It is utilized to choose TP_Routing_Instances from the same group that have not yet been included or less included than other instances. Every $T P \_$Routing_Instance having Type $_{\mathrm{B}}$-conflict is assigned an "Include Times" value " 0 " that gets incremeted whenever that particular TP_Routing_Instance becomes part of a generated combination.

In order to avoid combinations having Type $_{\mathrm{B}}$-conflict, the Rotate () function is called. Each time the function is called, it assigns a suitable value for parameter Token based on Type $\mathrm{B}_{\mathrm{B}}$-conflicts and the value of parameter Include Times. After calling the Rotate () function, Type $_{\mathrm{B}}$-conflicting TP_Routing_Instances with the minimum value of parameter Include Times will be assigned the value 1 for Token and all other Type B $_{\mathrm{B}}$-conflicting TP_Routing_Instances will get the value 2. Type B $_{\mathrm{B}}$-conflict-free TP_Routing_Instances will be assigned the value 0 . The algorithm for generating the final reduced combinations will avoid TP_Routing_Instances with the value 2 for Token parameter.

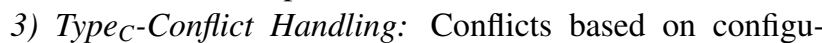
ration parameters are constraints considered in the test case selection and generation procedure. These constraints shall be corrected if error arises, e.g., correcting the maximum number of parallel routing instances of CAN transport protocol.

\section{Reduction of Test Suite}

In order to reduce the size of test suite, a new recursive test mechanism consisting of the following two test phases is proposed:

1) Testing TP parallel routing of Single Network Relationships (SNRs). An individual SNR comprises groups of TP_Routing_Instances responsible for routing TP data between two specific networks of the gateway. 
2) Testing TP parallel routing of Mixed Network Relationships (MNRs). An individual MNR comprises groups of $T P \_$Routing_Instances from all SNRs belonging to the same sub-IPM.

In the first test phase, testing of $\mathrm{TP}$ parallel routing for individual pairs of connected networks is carried out by means of groups belonging to individual SNRs. The goal of this test is to cover simple interactions (routing) between network pairs. After completing the first test phase, the second test phase is conducted. The goal of the second test phase is to cover complex interactions between multiple interacting networks by means of groups belonging to individual MNRs. Testing SNRs and MNRs separately is very useful for managing the test complexity and reducing the number of combinations. Furthermore, it provides information about possible reason for the occurrence of errors and their relationship with certain pairs of networks or parameter.

\section{A. Coverage Criterion}

Coverage criterion is an essential element of combination test strategies. It affects the complexity and the thoroughness of the test. In the proposed approach for testing TP parallel routing, coverage criteria is determined with respect to combinations of TP_Routing_Instances from constructed groups. For example, 1 -wise coverage requires that at least one possible combination with maximum allowed TP_Routing_Instances from every input group is included at least once in a test case. Since all TP_Routing_Instances of a group stimulate a similar behavior in the gateway, any one of such a combination from each group is sufficient enough to satisfy 1 -wise coverage criterion. $\mathrm{N}$-wise coverage criterion (exhaustive testing) requires that all possible combinations of TP_Routing_Instances from $N$ input groups are included in some test cases, where $N$ is the number of input groups.

For an arbitrary number of input groups, the algorithm generates all possible combinations satisfying the $\mathrm{N}$-wise interactions between the groups. The maximum number of resulting combinations is calculated as in (6):

$$
X=\frac{(n+y-1) !}{y !(n-1) !}
$$

Where $n$ is the number of groups and $y$ is the maximum number of allowed parallel TP_Routing_Instances. Table I explains the algorithm for generating combinations with the help of an example of 4 input groups $\left(\mathrm{G}_{1}, \mathrm{G}_{2}, \mathrm{G}_{3}, \mathrm{G}_{4}\right)$ and a maximum of 3 allowed parallel TP_Routing_Instances. Resulting rows in Table. I represent the combinations and the numbers in columns represent the number of $T P \_$Routing_Instances selected from each corresponding group for test case generation. The maximum number of resulting combinations (rows) in this example can be calculated according to (6) and is equal to 20 .

While generating test cases, following aspects are considered:

- To guarantee that each TP_Routing_Instance is included at least once in some combinations, the Rotate () function is used. Upon calling this function, it generates new Token
TABLE I

COMBINATIONS FROM 4 GROUPS WITH MAXIMUM OF 3 TP Routing Instances

\begin{tabular}{|l|c|c|c|}
\hline $\mathbf{G}_{\mathbf{1}}$ & $\mathbf{G}_{\mathbf{2}}$ & $\mathbf{G}_{\mathbf{3}}$ & $\mathbf{G}_{\mathbf{4}}$ \\
\hline 3 & 0 & 0 & 0 \\
\hline 2 & 1 & 0 & 0 \\
\hline 2 & 0 & 1 & 0 \\
\hline 2 & 0 & 0 & 1 \\
\hline 1 & 2 & 0 & 0 \\
\hline 1 & 1 & 1 & 0 \\
\hline 1 & 1 & 0 & 1 \\
\hline 1 & 0 & 2 & 0 \\
\hline 1 & 0 & 1 & 1 \\
\hline 1 & 0 & 0 & 2 \\
\hline 0 & 3 & 0 & 0 \\
\hline 0 & 2 & 1 & 0 \\
\hline 0 & 2 & 0 & 1 \\
\hline 0 & 1 & 2 & 0 \\
\hline 0 & 1 & 1 & 1 \\
\hline 0 & 1 & 0 & 2 \\
\hline 0 & 0 & 3 & 0 \\
\hline 0 & 0 & 2 & 1 \\
\hline 0 & 0 & 1 & 2 \\
\hline 0 & 0 & 0 & 3 \\
\hline & & & \\
\hline
\end{tabular}

values for TP_Routing_Instances based on the last values of Include Times variables and Type ${ }_{\mathrm{B}}$-Conflicts.

- The sum of column elements must be greater than or equal to the number of elements in the corresponding group in order to guarantee that each $T P \_$Routing_Instance has been included at least once. If this is not the case, the remaining TP_Routing_Instances from that group shall be tested individually.

- The number in each column must be lesser than or equal to the total number of TP_Routing_Instances in the corresponding group. Otherwise, the combination in the related row is invalid and must be omitted.

\section{B. TP Parallel Routing of SNRs}

The procedure for testing TP parallel routing of SNRs is depicted in Fig. 2. The procedure accepts constructed Type $_{\mathrm{A}^{-}}$ Conflict-free sub-IPMs as input and processes them successively. For each $\mathrm{Type}_{\mathrm{A}}$-Conflict-free sub-IPM, the Selector () function extracts groups for the first SNR. In the next step, a combination is built for the current processed SNR with the help of the mechanism explained previously satisfying $N$-wise coverage criterion. After that, a test case will be generated and executed for the build combination. During test case generation, Type $\mathrm{B}_{\mathrm{B}}$ - and $\mathrm{Type}_{\mathrm{C}}$-conflicts are handled as explained previously. Result of the test case is analyzed in the following step in which Stress Factor is determined and configuration parameters are corrected if a variance has been observed. This procedure is repeated for all combinations until the $N$-wise coverage criteria is satisfied for each SNR. This 


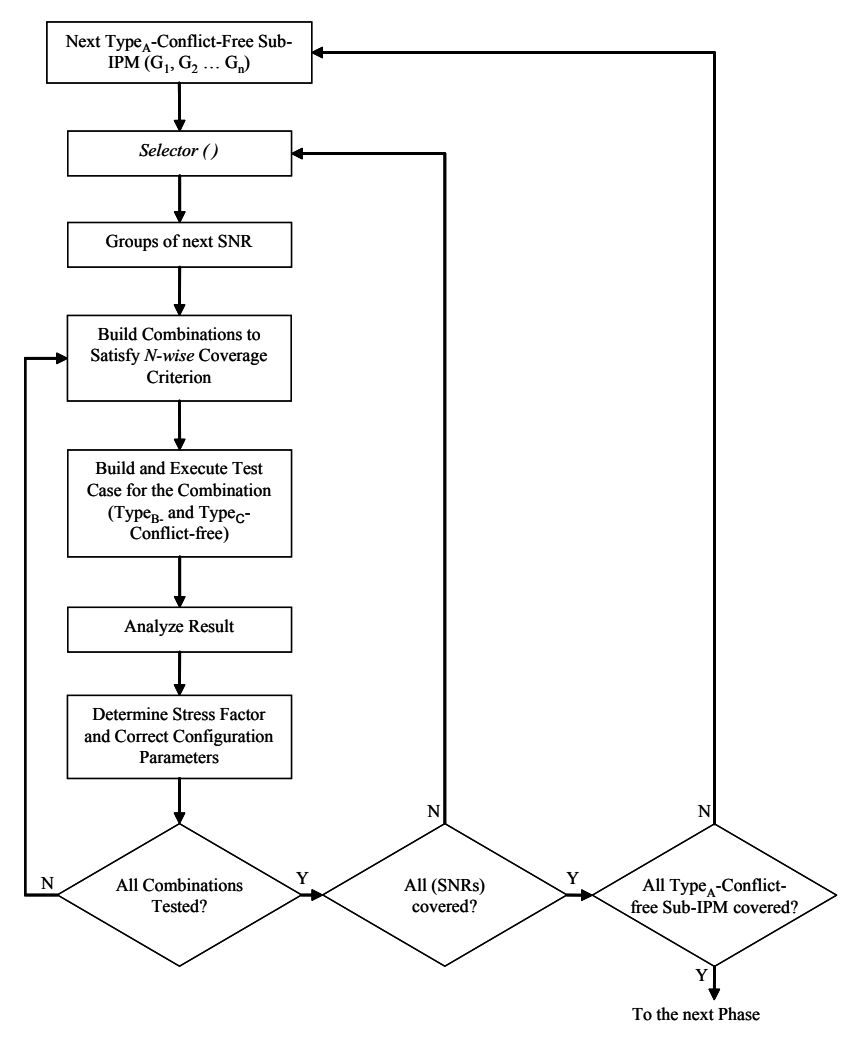

Fig. 2. TP Parallel Routing for SNRs

process is then repeated until all Type ${ }_{\mathrm{A}}$-conflict-free sub-IPM are covered. Processing combinations successively has the advantage to reduce further invalid combinations depending on the corrected configuration parameters. After finishing this phase, each group will be assigned a calculated Stress Factor, which will be used by the next phase.

\section{TP Parallel Routing of MNRs}

The procedure for testing TP parallel routing of MNRs is depicted in Fig. 3. In this procedure, Type ${ }_{\mathrm{A}}$-Conflict-free

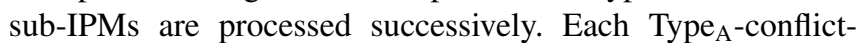
free sub-IPM consists of an arbitrary number of SNRs. A representative group with the best calculated Stress Factor for each of these SNR is selected. These representative groups are called MNR and they are the base for testing in this phase. That is, for each processed Type $_{\mathrm{A}}$-Conflict-free sub-IPM, a MNR with selected groups is constructed. After selecting groups of a MNR, groups having the same Similarity Numbers will be omitted in oder to reduce repititions in combinations. Then, the same procedure as that in previous phase is utilized to build combinations and execute test cases until $\mathrm{N}$-wise coverage criterion is satisfied. The procedure will stop once all Type $_{\mathrm{A}}$-Conflict-free sub-IPMs are processed. During recursive testing, constraints are corrected if an error is observed. This helps in further reduction of remaining combinations required to satisfy defined coverage criteria.

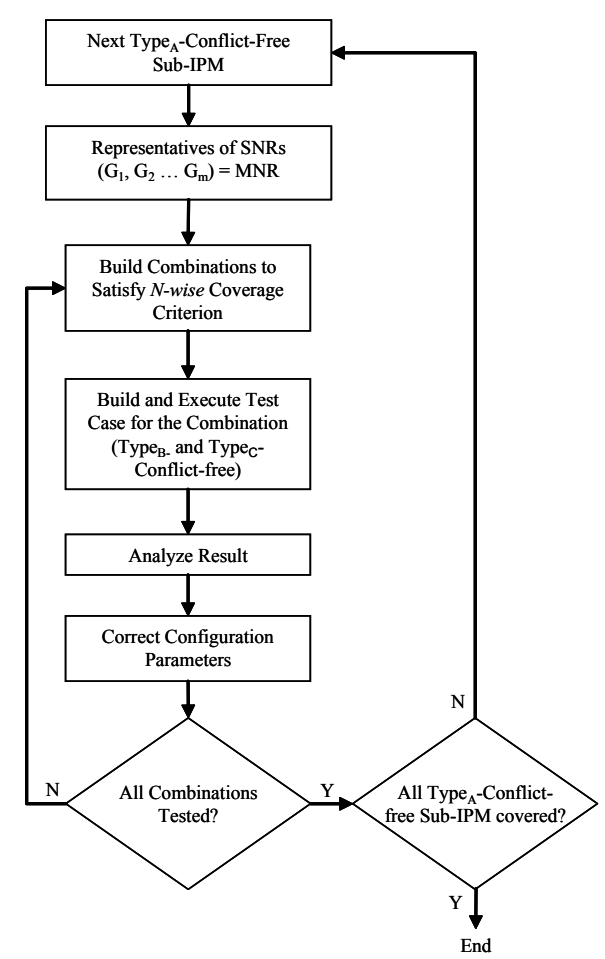

Fig. 3. TP Parallel Routing for MNRs

\section{EXPERIMENT}

The central gateway used in this experiment is a special electronic control unit which connects five different networks representing five functional domains of a modern vehicle. Bus systems of the five networks are listed as follows:

- A CAN network for diagnostic functional domain with 500 kilo baud, denoted as bus 1 .

- A CAN network for periphery and power train functional domain with 500 kilo baud, denoted as bus 2 .

- A CAN network for body functional domain with 250 kilo baud, denoted as bus 3 .

- A CAN network for telematics functional domain with 500 kilo baud, denoted as bus 4.

- A FlexRay network for chassis functional domain with $10 \mathrm{MB}$, denoted as bus 5 .

The gateway has 390 TP_Connection_Channels which are separated in:

- 190 TP_Connection_Channels used to transfer data from external device to available ECUs over the gateway.

- 190 TP_Connection_Channels used to transfer data from available ECUs to external device over the gateway.

- 4 TP_Connection_Channels used to transfer data between 4 couples of ECUs in the one direction.

- 4 TP_Connection_Channels used to transfer data between 4 couples of ECUs in the another direction.

- 2 functional TP_Connection_Channels.

Similarity criteria used to form groups are: 
- Network relationship of TP_Connection_Channels (source and destination networks).

- Addressing format (normal or mixed).

- Block Size (BS) and separation minimum time (STmin) if they are available.

- Cycle Repetition if it is available.

- ID Type (normal or extended).

- Address Type (physical or functional or extended).

- TP_Routing_Scenario

TP_Routing_Scenarios determined are:

- Flashing $\left(\mathrm{S}_{1}\right)$.

- Uploading $\left(\mathrm{S}_{2}\right)$.

- OBD in the one direction $\left(\mathrm{S}_{3}\right)$.

- OBD in the other direction $\left(\mathrm{S}_{4}\right)$.

- Functional routing $\left(\mathrm{S}_{5}\right)$.

Table II, table III, table IV and table V represent the resulting sub-IPMs with their corresponding groups.

Determined conflicts are listed below:

- Type $_{\mathrm{A}}$-Conflicts

- Flashing and Upload are non-combinable

- Flashing and OBD in both directions are noncombinable

- Upload and OBD in both directions are noncombinable

- OBD in both directions are non-combinable

- Type $_{\mathrm{B}}$-Conflicts

- TP_Connection_Channels with the same ID but different value for Node Address for Diagnose (NAD) are non-combinable

- TP_Connection_Channels with the same Slot-ID, Base-Cycle and Cycle-Repetition but different source address are non-combinable

- Type $_{C^{-}}$Conflicts

- The maximum configured parallel routing instances for CAN-CAN routing $<=30$

- The maximum configured parallel routing instances for CAN-FlexRay routing $<=8$

Transport protocols implemented are based on the ISO standards, ISO 10681 for FlexRay TP [11], and ISO 15765 for CAN TP [7]. Handling Type $_{B^{-}}$and Type ${ }_{C}-$ Conflicts during test lead to following maximum number of combinations in the first test phase:

- First sub-IPM

- First SNR: 10 combinations

- Second SNR: 152 combinations

- Third SNR: 1 combination

- Fourth SNR: 31 combinations

- Second sub-IPM

- First SNR: 10 combinations

- Second SNR: 78 combinations

- Third SNR: 1 combination

- Fourth SNR: 31 combinations

- Third sub-IPM
- First SNR: 1 combination

- Second SNR: 1 combination

- Third SNR: 1 combination

- Fourth SNR: 1 combination

- Fourth sub-IPM

- First SNR: 1 combination

- Second SNR: 1 combination

- Third SNR: 1 combination

- Fourth SNR: 1 combination

Table VI represents an example of a resulting combination from the first SNR of the first Sub-IPM. The first SNR of the first Sub-IPM consists of the groups $G_{1}, G_{2}$, $\mathrm{G}_{3}, \mathrm{G}_{4}, \mathrm{G}_{18}$ and $\mathrm{G}_{19}$ from table II. Following numbers of $T P \_$Routing_Instances have been selected to generate the first combination:

- 5 TP_Routing_Instances from group $\mathrm{G}_{1}$

- 8 TP_Routing_Instances from group $\mathrm{G}_{2}$

- 14 TP_Routing_Instances from group $\mathrm{G}_{3}$

- 3 TP_Routing_Instances from group $\mathrm{G}_{4}$

\section{DISCUSSION}

Depending on the grade of diversity in parameters for TP_Connection_Channels and in connected networks; the number of formulated groups can increase. The idea is to cover the interactions between formulated groups instead of interactions between TP_Routing_Instances. This helps in avoiding similar combinations and contribute to reduce the size of the test suit. If the number $N$ of groups is high, either a 2-Wise or 3 -wise coverage criteria can be used. A drawback of proposed approach is the need of system functionality expertise to define similarity criterion and calculate the Stress Factors of the groups. However this needs to be performed only once. Later on, for each new release of the system, combinations can be generated automatically.

\section{CONCLUSION AND FUtURE WORK}

In this paper, an approach for building a conflict-free IPM to test TP parallel routing on a vehicle gateway system has been proposed. Firstly, the approach utilizes defined similarity criteria to cluster system input parameters represented as TP_Routing_Instances into groups stimulating similar behavior. Secondly, the two conflict handling methods submodels and avoid are used to prohibit invalid combinations of TP_Routing_Instances. In order to reduce the size of the test suite, two phases of testing have been suggested, testing TP parallel routing for SNRs in order to cover simple interactions between network pairs, and testing TP parallel routing for MNRs to cover complex interactions between multiple interacting networks. The proposed approach has been applied on a complex example of a gateway with five buses, 390 TP_Routing_Instances and diverse conflicts. Generating test cases for resulting combinations from the first test phase and conducting the second test phase will be achieved in the future. Furthermore, a restbus simulation is under development to execute the generated test cases for build combinations. 
TABLE II

FIRST SUB-IPM's GROUPS

\begin{tabular}{|c|c|c|c|c|c|c|c|}
\hline Groups & $\begin{array}{c}\text { Network } \\
\text { Relationship }\end{array}$ & $\begin{array}{c}\text { Addressing } \\
\text { Format }\end{array}$ & BS and STmin & $\begin{array}{c}\text { Cycle } \\
\text { Repetition }\end{array}$ & ID Type & $\begin{array}{c}\text { Address } \\
\text { Type }\end{array}$ & $\begin{array}{l}\text { TP Routing } \\
\text { Scenario }\end{array}$ \\
\hline $\mathrm{G}_{1}$ (19 elements) & 1,2 & Mixed & $(32,0)(32,0)$ & - & Normal & Physical & $\mathrm{S}_{1}$ \\
\hline $\mathrm{G}_{2}$ (8 elements) & 1,2 & Normal & $(32,0)(8,10)$ & - & Norma & Physicall & $\mathrm{S}_{1}$ \\
\hline $\mathrm{G}_{3}$ (14 elements) & 1,2 & Normal & $(32,0)(32,0)$ & - & Normal & Physical & $\mathrm{S}_{1}$ \\
\hline $\mathrm{G}_{4}$ (8 elements) & 1,2 & Normal & $(8,10)(8,10)$ & - & Extended & Physical & $\mathrm{S}_{1}$ \\
\hline $\mathrm{G}_{5}$ (58 elements) & 1,3 & Mixed & $(32,0)(32,0)$ & - & Normal & Physical & $\mathrm{S}_{1}$ \\
\hline $\mathrm{G}_{7}$ (5 elements) & 1,3 & Mixed & $(32,0)(32,20)$ & - & Normal & Physical & $\mathrm{S}_{1}$ \\
\hline $\mathrm{G}_{8}$ (3 elements) & 1,3 & Normal & $(32,0)(32,0)$ & - & Normal & Physical & $\mathrm{S}_{1}$ \\
\hline $\mathrm{G}_{9}$ (8 elements) & 1,4 & Normal & $(32,0)(32,0)$ & - & Normal & Physical & $\mathrm{S}_{1}$ \\
\hline $\mathrm{G}_{10}(2$ elements $)$ & 1,4 & Normal & $(4,10)(4,10)$ & - & Normal & Physical & $\mathrm{S}_{1}$ \\
\hline $\mathrm{G}_{11}$ (13 elements) & 1,4 & Normal & $(32,0)(8,10)$ & - & Normal & Physical & $\mathrm{S}_{1}$ \\
\hline $\mathrm{G}_{12}$ (5 elements) & 1,4 & Mixed & $(32,0)(32,0)$ & - & Normal & Physical & $\mathrm{S}_{1}$ \\
\hline $\mathrm{G}_{14}$ (1 elements) & 1,5 & Normal & $(32,0)(-,-)$ & 1 & Normal & Physical & $\mathrm{S}_{1}$ \\
\hline $\mathrm{G}_{15}$ (1 elements) & 1,5 & Mixed & $(32,0)(-,-)$ & 4 & Normal & Physical & $\mathrm{S}_{1}$ \\
\hline $\mathrm{G}_{16}$ (1 elements) & 1,5 & Normal & $(8,10)(-,-)$ & 1 & Extended & Physical & $\mathrm{S}_{1}$ \\
\hline $\mathrm{G}_{17}$ (7 elements) & 1,5 & Normal & $(32,0)(-,-)$ & 4 & Normal & Physical & $\mathrm{S}_{1}$ \\
\hline $\mathrm{G}_{18}$ (1 elements) & $1,(2,3,4,5)$ & Normal & $(-,-)(-,-)$ & - & Normal & Functional & $\mathrm{S}_{5}$ \\
\hline $\mathrm{G}_{19}$ (1 elements) & $1,(2,5)$ & Normal & $(-,-)(-,-)$ & - & Extended & Functional & $\mathrm{S}_{5}$ \\
\hline
\end{tabular}

TABLE III

SECOND SUB-IPM'S GRouPS

\begin{tabular}{|c|c|c|c|c|c|c|c|}
\hline Groups & $\begin{array}{c}\text { Network } \\
\text { Relationship }\end{array}$ & $\begin{array}{c}\text { Addressing } \\
\text { Format }\end{array}$ & BS and STmin & $\begin{array}{c}\text { Cycle } \\
\text { Repetition }\end{array}$ & ID Type & $\begin{array}{l}\text { Address } \\
\text { Type }\end{array}$ & $\begin{array}{c}\text { TP Routing } \\
\text { Scenario }\end{array}$ \\
\hline $\mathrm{G}_{1}$ (15 elements) & 2,1 & Normal & $(8,0)(8,0)$ & - & Normal & Physical & $\mathrm{S}_{2}$ \\
\hline $\mathrm{G}_{2}$ (7 elements) & 2,1 & Normal & $(8,10)(8,0)$ & - & Normal & Physical & $\mathrm{S}_{2}$ \\
\hline $\mathrm{G}_{3}$ (19 elements) & 2,1 & Mixed & $(8,0)(8,0)$ & - & Normal & Physical & $\mathrm{S}_{2}$ \\
\hline $\mathrm{G}_{4}$ (8 elements) & 2,1 & Normal & $(8,0)(8,0)$ & - & Extended & Physical & $\mathrm{S}_{2}$ \\
\hline $\mathrm{G}_{5}$ (63 elements) & 3,1 & Mixed & $(8,0)(8,0)$ & - & Normal & Physical & $\mathrm{S}_{2}$ \\
\hline $\mathrm{G}_{7}$ (3 elements) & 3,1 & Normal & $(8,0)(8,0)$ & - & Normal & Physical & $\mathrm{S}_{2}$ \\
\hline $\mathrm{G}_{8}$ (8 elements) & 4,1 & Normal & $(8,0)(8,0)$ & - & Normal & Physical & $\mathrm{S}_{2}$ \\
\hline G9 (13 elements) & 4,1 & Normal & $(8,10)(8,0)$ & - & Normal & Physical & $\mathrm{S}_{2}$ \\
\hline $\mathrm{G}_{10}$ (5 elements) & 4,1 & Mixed & $(8,0)(8,0)$ & - & Normal & Physical & $\mathrm{S}_{2}$ \\
\hline $\mathrm{G}_{11}$ (2 elements) & 4,1 & Normal & $(4,10)(4,10)$ & - & Normal & Physical & $\mathrm{S}_{2}$ \\
\hline $\mathrm{G}_{12}$ (8 elements) & 5,1 & Normal & $(-,-)(8,0)$ & 2 & Normal & Physical & $\mathrm{S}_{2}$ \\
\hline $\mathrm{G}_{14}$ (7 elements) & 5,1 & Normal & $(-,-)(8,0)$ & 4 & Normal & Physical & $\mathrm{S}_{2}$ \\
\hline $\mathrm{G}_{15}$ (1 elements) & 5,1 & Mixed & $(-,-)(8,0)$ & 4 & Normal & Physical & $\mathrm{S}_{2}$ \\
\hline $\mathrm{G}_{16}$ (1 elements) & 5,1 & Normal & $(-,-)(8,0)$ & 1 & Extended & Physical & $\mathrm{S}_{2}$ \\
\hline $\mathrm{G}_{17}$ (1 elements) & $1,(2,3,4,5)$ & Normal & $(-,-)(-,-)$ & - & Normal & Functional & $\mathrm{S}_{5}$ \\
\hline $\mathrm{G}_{18}$ (1 elements) & $1,(2,5)$ & Normal & $(-,-)(-,-)$ & - & Extended & Functional & $\mathrm{S}_{5}$ \\
\hline
\end{tabular}


TABLE IV

THIRD SUB-IPM'S GROUPS

\begin{tabular}{|l|c|l|c|c|c|c|c|}
\hline \multicolumn{1}{|c|}{ Groups } & $\begin{array}{c}\text { Network } \\
\text { Relationship }\end{array}$ & \multicolumn{1}{|c|}{$\begin{array}{c}\text { Addressing } \\
\text { Format }\end{array}$} & BS and STmin & $\begin{array}{c}\text { Cycle } \\
\text { Repetition }\end{array}$ & ID Type & $\begin{array}{c}\text { Address } \\
\text { Type }\end{array}$ & $\begin{array}{c}\text { TP Routing } \\
\text { Scenario }\end{array}$ \\
\hline $\mathrm{G}_{1}(3$ elements $)$ & 3,4 & Normal & $(4,10)(4,10)$ & - & Normal & Physical & $\mathrm{S}_{3}$ \\
\hline $\mathrm{G}_{2}(1$ elements $)$ & 4,5 & Normal & $(4,20)(-,-)$ & 16 & Normal & Physical & $\mathrm{S}_{3}$ \\
\hline $\mathrm{G}_{3}(1$ elements $)$ & $1,(2,3,4,5)$ & Normal & $(-,-)(-,-)$ & - & Normal & Functional & $\mathrm{S}_{5}$ \\
\hline $\mathrm{G}_{4}(1$ elements $)$ & $1,(2,5)$ & Normal & $(-,-)(-,-)$ & - & Extended & Functional & $\mathrm{S}_{5}$ \\
\hline
\end{tabular}

TABLE V

FORTH SUB-IPM'S GROUPS

\begin{tabular}{|l|c|l|c|c|c|c|c|}
\hline \multicolumn{1}{|c|}{ Groups } & $\begin{array}{c}\text { Network } \\
\text { Relationship }\end{array}$ & \multicolumn{1}{|c|}{$\begin{array}{c}\text { Addressing } \\
\text { Format }\end{array}$} & BS and STmin & $\begin{array}{c}\text { Cycle } \\
\text { Repetition }\end{array}$ & ID Type & $\begin{array}{c}\text { Address } \\
\text { Type }\end{array}$ & $\begin{array}{c}\text { TP Routing } \\
\text { Scenario }\end{array}$ \\
\hline $\mathrm{G}_{1}(3$ elements $)$ & 4,3 & Normal & $(4,10)(4,10)$ & - & Normal & Physical & $\mathrm{S}_{4}$ \\
\hline $\mathrm{G}_{2}(1$ elements $)$ & 5,4 & Normal & $(-,-)(8,20)$ & 16 & Normal & Physical & $\mathrm{S}_{4}$ \\
\hline $\mathrm{G}_{3}(1$ elements $)$ & $1,(2,3,4,5)$ & Normal & $(-,-)(-,-)$ & - & Normal & Functional & $\mathrm{S}_{5}$ \\
\hline $\mathrm{G}_{4}(1$ elements $)$ & $1,(2,5)$ & Normal & $(-,-)(-,-)$ & - & Extended & Functional & $\mathrm{S}_{5}$ \\
\hline
\end{tabular}

TABLE VI

An Example of a Resulting Combination

\begin{tabular}{|c|c|c|c|c|c|c|c|c|}
\hline Request ID & Response ID & $\begin{array}{c}\text { Network } \\
\text { Relationship }\end{array}$ & BS and STmin & $\begin{array}{c}\text { Message } \\
\text { DLC }\end{array}$ & NAD & $\begin{array}{c}\text { Addressing } \\
\text { Format }\end{array}$ & ID Type & $\begin{array}{c}\text { TP Routing } \\
\text { Scenario }\end{array}$ \\
\hline $0 \times 4 \mathrm{e} 9$ & 0x499 & 1,2 & $(32,0)(32,0)$ & 8 & 14 & Mixed & Normal & $S_{1}$ \\
\hline $0 \times 4 \mathrm{e} 8$ & $0 \times 498$ & 1,2 & $(32,0)(32,0)$ & 8 & 32 & Mixed & Normal & $\mathrm{S}_{1}$ \\
\hline $0 \times 4 \mathrm{c} 4$ & $0 \times 494$ & 1,2 & $(32,0)(32,0)$ & 8 & 5 & Mixed & Normal & $\mathrm{S}_{1}$ \\
\hline $0 \mathrm{x} 4 \mathrm{~d} 0$ & $0 \times 490$ & 1,2 & $(32,0)(32,0)$ & 8 & 8 & Mixed & Normal & $S_{1}$ \\
\hline $0 \mathrm{x} 4 \mathrm{e} 7$ & $0 \times 497$ & 1,2 & $(32,0)(32,0)$ & 8 & 13 & Mixed & Normal & $S_{1}$ \\
\hline $0 \times 450$ & $0 \times 5 \mathrm{~d} 9$ & 1,2 & $(32,0)(8,10)$ & 8 & - & Normal & Normal & $S_{1}$ \\
\hline $0 \times 456$ & $0 \times 5 \mathrm{~d} 5$ & 1,2 & $(32,0)(8,10)$ & 8 & - & Normal & Normal & $S_{1}$ \\
\hline $0 \times 7 e 4$ & $0 \times 7 \mathrm{ec}$ & 1,2 & $(32,0)(8,10)$ & 8 & - & Normal & Normal & $S_{1}$ \\
\hline $0 \times 625$ & $0 \times 5 a 5$ & 1,2 & $(32,0)(8,10)$ & 8 & - & Normal & Normal & $\mathrm{S}_{1}$ \\
\hline $0 \times 654$ & $0 \times 5 \mathrm{~d} 4$ & 1,2 & $(32,0)(8,10)$ & 8 & - & Normal & Normal & $\mathrm{S}_{1}$ \\
\hline $0 \times 652$ & $0 \times 5 \mathrm{~d} 2$ & 1,2 & $(32,0)(8,10)$ & 8 & - & Normal & Normal & $\mathrm{S}_{1}$ \\
\hline $0 \times 624$ & $0 \times 5 \mathrm{a} 4$ & 1,2 & $(32,0)(8,10)$ & 8 & - & Normal & Normal & $\mathrm{S}_{1}$ \\
\hline $0 \times 653$ & $0 \times 5 \mathrm{~d} 3$ & 1,2 & $(32,0)(8,10)$ & 8 & - & Normal & Normal & $\mathrm{S}_{1}$ \\
\hline $0 \times 64 \mathrm{e}$ & $0 \times 5 \mathrm{ce}$ & 1,2 & $(32,0)(32,0)$ & 8 & - & Normal & Normal & $\mathrm{S}_{1}$ \\
\hline $0 \times 7 \mathrm{e} 5$ & 0x7ed & 1,2 & $(32,0)(32,0)$ & 8 & - & Normal & Normal & $\mathrm{S}_{1}$ \\
\hline $0 \times 7 \mathrm{e} 1$ & 0x7ea & 1,2 & $(32,0)(32,0)$ & 8 & - & Normal & Normal & $S_{1}$ \\
\hline $0 \times 665$ & $0 \times 5 \mathrm{e} 5$ & 1,2 & $(32,0)(32,0)$ & 8 & - & Normal & Normal & $S_{1}$ \\
\hline $0 \times 778$ & $0 \times 788$ & 1,2 & $(32,0)(32,0)$ & 8 & - & Normal & Normal & $S_{1}$ \\
\hline $0 x 64 d$ & $0 \times 5 \mathrm{~cd}$ & 1,2 & $(32,0)(32,0)$ & 8 & - & Normal & Normal & $S_{1}$ \\
\hline $0 \times 656$ & $0 \times 5 \mathrm{~d} 6$ & 1,2 & $(32,0)(32,0)$ & 8 & - & Normal & Normal & $\mathrm{S}_{1}$ \\
\hline $0 \times 650$ & $0 \times 5 \mathrm{~d} 0$ & 1,2 & $(32,0)(32,0)$ & 8 & - & Normal & Normal & $S_{1}$ \\
\hline $0 \times 7 \mathrm{e} 2$ & $0 \times 7 \mathrm{e} 8$ & 1,2 & $(32,0)(32,0)$ & 8 & - & Normal & Normal & $\mathrm{S}_{1}$ \\
\hline $0 \times 7 \mathrm{e} 6$ & $0 \times 7$ ee & 1,2 & $(32,0)(32,0)$ & 8 & - & Normal & Normal & $S_{1}$ \\
\hline $0 \times 7 d 9$ & $0 \times 7 \mathrm{e} 7$ & 1,2 & $(32,0)(32,0)$ & 8 & - & Normal & Normal & $S_{1}$ \\
\hline $0 \times 7 \mathrm{e} 3$ & $0 \times 7 \mathrm{eb}$ & 1,2 & $(32,0)(32,0)$ & 8 & - & Normal & Normal & $S_{1}$ \\
\hline $0 \times 662$ & $0 \times 5 \mathrm{e} 2$ & 1,2 & $(32,0)(32,0)$ & 8 & - & Normal & Normal & $S_{1}$ \\
\hline $0 \times 65 b$ & $0 \times 6 \mathrm{db}$ & 1,2 & $(32,0)(32,0)$ & 8 & - & Normal & Normal & $S_{1}$ \\
\hline 0x18da69f1 & 0x18daf169 & 1,2 & $(8,10)(8,10)$ & 8 & - & Normal & Extended & $S_{1}$ \\
\hline 0x18da66f1 & 0x18daf166 & 1,2 & $(8,10)(8,10)$ & 8 & - & Normal & Extended & $S_{1}$ \\
\hline 0x18da43f1 & 0x18daf143 & 1,2 & $(8,10)(8,10)$ & 8 & - & Normal & Extended & $\mathrm{S}_{1}$ \\
\hline
\end{tabular}




\section{REFERENCES}

[1] M. Grindal, J. Offutt, and S. F. Andler, "Combination Testing Strategies: A survey," GMU Technical Report ISE-TR-04-05, July 2004.

[2] M.N. Borazjany, L. S. Ghandehari, Y. Lei, R.N. Kacker, and D.R. Kuhn, "An Input Space Modeling Methodology for Combinatorial Testing," Software Testing, Verification and Validation Workshops (ICSTW), 2013 IEEE Sixth International Conference, pp. 372-381, Luxembourg 2013.

[3] M. Grindal and J. Offutt, "Input Parameter Modeling for Combination Strategies," In Proceeding SE'07 Proceedings of the 25th conference on IASTED International Multi-Conference: Software Engineering, pp. 255-260, USA 2007.

[4] M. Grindal, J. Offutt, and J. Mellin, "Handling Constraints in the Input Space when Using Combination Strategies for Software Testing," Technical Report HS-IKI-TR-06-001. School of Humanities and Informatics, University of Skövde 2006.

[5] S. A Vilkomir, W. T. Swain, and J. H. Poore, "Software Input Space Modeling with Constraints among Parameters," Computer Software and Applications Conference, COMPSAC '09. 33rd Annual IEEE International, pp. 136-141, Seattle. WA. 2009.
[6] M. Grindal, J. Offutt, and J. Mellin, "Managing Conflicts when Using Combination Strategies to Test Software," Software Engineering Conference, ASWEC 2007. 18th Australian, pp. 255-264. Melbourne, Vic. 2007.

[7] "Road vehicles-Diagnostics on Controller Area Networks (CAN)-," ISO 15765:2004(E), Switzerland : ISO.

[8] D. M. Cohen, S. R. Dalal, M. L. Fredman, and G. C. Patton, "The AETG System: An Approach to Testing Based on Combinatorial Design," IEEE Transaction on Software Engineering, vol. 23 ,pp. 437-444, July 1997.

[9] R. Mandl, "Orthogonal Latin Squares: An application of experiment design to compiler testing," Communications of the ACM, 28(10):1054 -1058, October 1985

[10] C. J. Colbourn, M. B. Cohen, and R. C. Turban, "A Deterministic Density Algorithm for Pairwise Interaction Coverage," In: Proc. of the IASTED Intl. Conference on Software Engineering, pp. 345-352, Austria 2004.

[11] "Road vehicles-Communication on FlexRay-," ISO 10681:2010(E), Switzerland : ISO. 